\title{
The role of experimenter-odor cues in the performance of object-memory tasks by rats
}

\author{
DAVE G. MUMBY, TOM J. KORNECOOK, EMMA R. WOOD, and JOHN P. J. PINEL \\ University of British Columbia, Vancouver, British Columbia, Canada
}

\begin{abstract}
Two experiments assessed whether odors left on stimulus objects by experimenters who handle them might confound the interpretation of ostensibly visually guided object-memory tasks for rats. In Experiment 1, rats were able to discriminate the relative recency with which an experimenter touched two otherwise identical objects (intertouch interval $=4 \mathrm{sec}$ ), presumably on the basis of an odorintensity discrimination. However, after the rats mastered the odor discrimination with no delay between when the second of the two stimulus objects was last touched by the experimenter and when the rats were permitted to attempt the discrimination, their performance dropped to chance levels when this delay was increased to $15 \mathrm{sec}$. In Experiment 2, rats were trained in two slightly different ways to perform a delayed-nonmatching-to-sample (DNMS) task, one that involved systematic differences in the temporal order in which the experimenter handled the sample and novel stimulus objects and one that did not. There were no significant differences in the rate at which rats mastered the DNMS task with these two procedures, and the performance of rats that were trained according to the former procedure was unaffected when they were switched to the latter procedure. Moreover, rats required considerably fewer trials to master the DNMS task than the rats in Experiment 1 required to master the odor discrimination. These findings demonstrate that, under certain circumstances, rats can discriminate the relative recency with which two objects are handled by an experimenter, but that this ability contributes little to their performance of conventional object-based DNMS tasks.
\end{abstract}

Studies of amnesia in monkeys commonly employ object-memory tasks, such as simple two-choice object discriminations, multiple-pair concurrent object discriminations, or nonrecurring item delayed nonmatching-to-sample or matching-to-sample (Zola-Morgan, Squire, \& Amaral, 1989). Monkeys perform these tasks by reaching out with one hand and moving the test object of their choice from a food well located several inches away (Mishkin \& Appenzeller, 1987). The objects are positioned too far away for monkeys to discriminate their olfactory features, and subjects do not palpate the objects prior to making a choice. Thus, monkeys appear to be responding primarily, if not totally, on the basis of the visual differences between objects.

We recently developed a battery of object-memory tasks for rats that resembles the test battery used in monkeys. The battery includes the following tasks: a simple two-choice object discrimination and discrimination reversal, an eight-pair concurrent object discrimination, and a nonrecurring-items delayed-nonmatching-to-sample (DNMS) task with various retention delays and sample-

This research was supported by a grant to J.P.J.P. from the British Columbia Health Care Research Foundation. D.G.M. was supported by a postgraduate studentship from the Medical Research Council of Canada. E.R.W. was supported by a Commonwealth Scholarship. The authors thank Gerard Martin and the two anonymous reviewers for their helpful comments on this manuscript, and Maggie Edwards for the drawing in Figure 1. Correspondence should be addressed to D. G. Mumby, who is now at the Department of Psychology, DS-413, Concordia University, 7141 Sherbrooke St. W., Montreal, Quebec, Canada H4B IR6. list lengths (Mumby, Pinel, \& Anzarut, 1991; Mumby, Pinel, Kornecook, Shen, \& Redila, 1995; Mumby, Pinel, $\&$ Wood, 1990). The rat versions of these tasks resemble their monkey counterparts in several key respects; for example, the test stimuli are objects, and, on each trial, the rat selects an object by displacing it from over a food well. In principle, these similarities should facilitate direct comparisons of object-memory abilities and their neural bases in rats and monkeys. However, despite these similarities among the rat and monkey tasks, rats may not solve objectmemory problems on the basis of visual differences between objects. Because rats displace objects with the snout (or, less frequently, with the forepaws), they have an opportunity to smell and feel the objects before making their selection.

In the present experiments, we examined the possibility that rats might use odor cues to solve the object-memory tasks in our test battery. Specifically, we were concerned with the possible confounding effect of odors left on stimulus objects by the experimenter, who must handle them on each trial. We focused our examination of this problem on the DNMS task because we have relied on this objectmemory task in most of our previous research (Mumby \& Pinel, 1994; Mumby, Pinel, \& Dastur, 1993; Mumby et al., 1995; Mumby et al., 1990; Mumby, Wood, \& Pinel, 1992; Wood, Mumby, Pinel, \& Phillips, 1993).

Our DNMS procedure is illustrated in Figure 1. A rat is enclosed in the central starting area prior to the start of each trial. Two objects, one that serves as the sample object and another that serves as the novel (nonsample) object on that trial, are positioned over food wells located at 
A

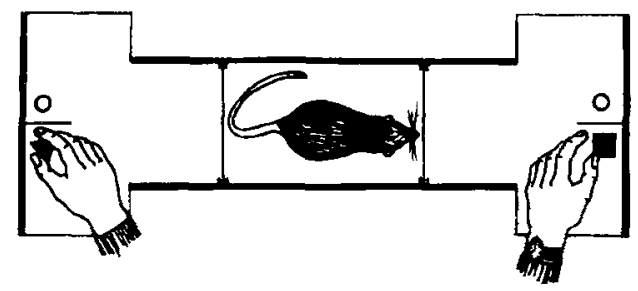

B

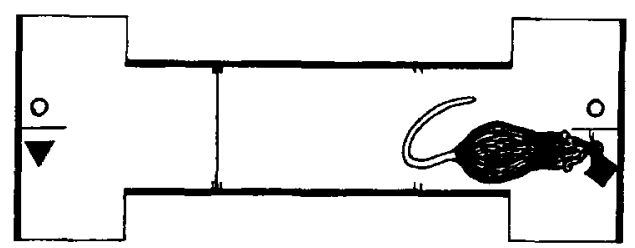

C

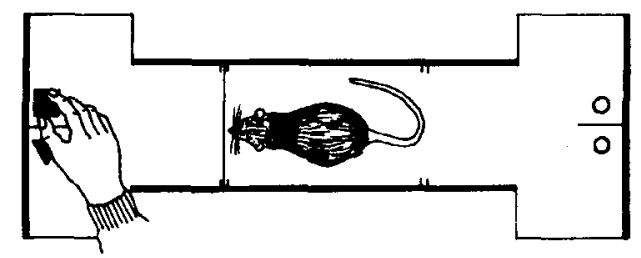

D

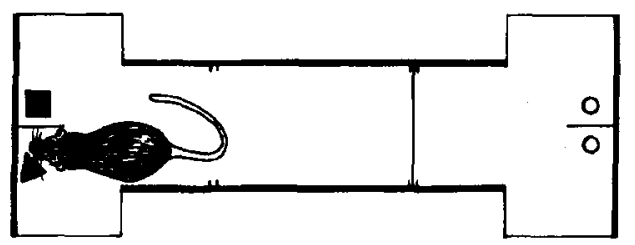

E

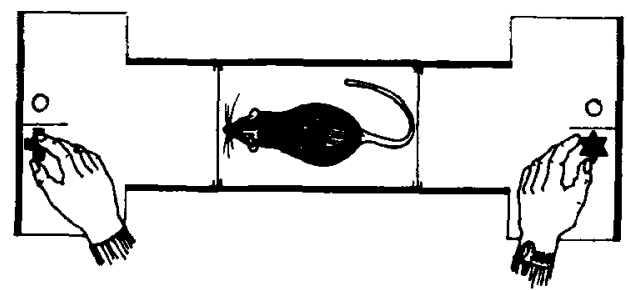

Figure 1. The DNMS training procedures originally described by Mumby et al. (1990). (A) Prior to each trial, the rat is enclosed in the central area while the experimenter positions the sample and novel objects over food wells at opposite ends of the apparatus. (B) The door is opened to allow access to the sample object, which the rat approaches and displaces from the well. (C) The experimenter immediately removes the sample object and positions it over the vacant well next to the novel object. (D) After a retention delay, the door is opened to allow access to both objects. The rat is rewarded if it displaces the novel object, but not if it displaces the sample object. (E) After it has made its choice, the rat returns to the central area, the doors are lowered, and new sample and novel objects are positioned in preparation for the next trial.

each end of the apparatus. A food pellet is hidden in the well beneath the sample object. The door at the sample end is opened, allowing the rat to approach and displace the sample object from over the food well so that it can retrieve the food pellet. The experimenter immediately removes the sample object and places it over the vacant food well at the other end of the apparatus, next to the novel ob- ject. Access to the goal area, which now contains both the sample and the novel object, is restricted until the end of the prescribed retention delay. The door is then opened so that the rat can approach and displace one of the two objects. If a rat displaces the novel object (the correct choice), a food pellet is delivered into the food well; if it displaces the sample object (the incorrect choice), no pellet is delivered. Different objects are used on each trial within a session.

When well-trained rats perform the DNMS task at high levels of accuracy, they tend to veer toward the correct object while they are approaching the goal area and still several centimeters away from the objects (see Mumby et al., 1990). This observation suggests that rats do not rely on tactual cues to solve the DNMS task. Furthermore, we routinely wash the objects every few sessions in a solution of chlorine bleach and water to reduce the rats' ability to discriminate between them on the basis of olfactory cues. Nonetheless, certain extraneous odors cannot be kept off the objects in this way. In particular, washing the objects cannot prevent the scent of the experimenter from getting onto them because it is necessary for the experimenter to handle the objects on every trial. During the test phase of a DNMS trial, the sample and the novel objects should have a trace of the experimenter's scent on them because the experimenter recently handled both of them, but the intensity of this scent might be consistently greater on the sample object because it is always handled more recently than the novel object (see Figure 1). Thus, rats might be able to solve the DNMS task on the basis of olfactory discrimination, rather than purely on the basis of visual discrimination. As unlikely as this possibility may seem, it cannot be ignored given the excellent performance by rats on odor-guided discrimination and DNMS tasks (e.g., Otto \& Eichenbaum, 1992).

In view of the fact that the rat DNMS task is presumed to model the primate DNMS task, it is important to clarify the role of olfaction in the rat task. The general purposes of the present experiments were (1) to determine whether rats could discriminate the relative recency with which two objects had been handled by an experimenter and (2) to determine whether rats use this ability to solve the DNMS task.

\section{EXPERIMENT 1 Odor Discrimination}

The purpose of Experiment 1 was to determine whether rats can discriminate the relative recency with which two otherwise identical objects were handled by an experimenter. If rats can perform such a discrimination, then precautions would be necessary to avoid this potential confound when testing rats' visual memory for objects.

\section{Method}

Subjects. Four experimentally naive male Long-Evans rats (Charles River, Quebec), which were between 7 and 9 weeks old at the beginning of the experiment, were housed individually with continuous access to water under a 12:12-h light:dark cycle with light onset at 8:00 a.m. The rats were reduced to and maintained at ap- 
proximately $85 \%$ of their free-feeding body weights throughout the experiment. Training began after the rats had been on the restrictedfeeding regimen for 14 days.

Apparatus. The apparatus shown in Figure 1 has been previously described in detail (Mumby et al., 1990). Briefly, the elevated runway is separated from identical goal areas at each end by opaque guillotine doors. Each goal area contains two food wells into which food pellets ( $45 \mathrm{mg}$, Bio Serv Inc., Frenchtown, NJ) can be delivered by hand through plastic tubes that are mounted on the outside of the apparatus. The two food wells are separated by a short divider wall $(9 \times 9 \mathrm{~cm})$ that protrudes from the center of the end wall. The sides of the goal areas are open to allow the experimenter to place stimulus objects over the food wells and to remove them quickly.

A set of 40 identical, orange-plastic bottle lids ( $7 \mathrm{~cm}$ in diameter) served as the stimulus objects. The lids were large enough to cover the food wells but light enough to be easily displaced by rats. All 40 lids were washed in a dilute solution of chlorine bleach and water after each session in order to eliminate extraneous scents.

Procedure. All testing occurred during the light phase of the light:dark cycle, between 15 and $22 \mathrm{~h}$ after a rat's most recent meal. Each rat was tested no more than once per day and no fewer than five times per week. The rats were not handled during a test session once they had been placed in the apparatus. After placing a rat in the apparatus, the experimenter rinsed his hands thoroughly with water (without soap) to lessen any scents that they might have acquired from having handled the rat.

The rats were habituated to the apparatus and shaped to retrieve food pellets from the wells (see Mumby et al., 1990) and were then trained to discriminate between objects on the basis of the relative recency with which they had been touched by the experimenter. At the beginning of each session, one of the doors to the goal area was open and the other door was closed. The food wells on the side of the apparatus with the open door were uncovered and unbaited. A rat was then placed in the center of the apparatus. One of 40 lids was placed over one of the food wells behind the closed door. This lid by virtue of having been touched first by the experimenter, was designated $\mathrm{S}+$. The choice of $\mathrm{S}+$ was rewarded. Approximately $4 \mathrm{sec}$ following the placement of $S+$, the experimenter placed a second lid over the vacant food well. This second lid was designated $\mathrm{S}-$. The choice of $\mathrm{S}-$ was not rewarded. The position of $\mathrm{S}+$ on the left or right food well varied across trials in a pseudorandom pattern. Thus, the only consistent difference between $\mathrm{S}+$ and $\mathrm{S}-$ was that the $\mathrm{S}+$ was always handled about $4 \mathrm{sec}$ before $S-$. An intertouch interval of $4 \mathrm{sec}$ was used because of its similarity to the interval between when sample and novel objects are touched by the experimenter in our DNMS procedures. Immediately after $S-$ was positioned, the experimenter opened the door to expose the two lids. When a rat approached and displaced one of the lids, the far door was lowered. The two lids were removed following a choice. Two other lids were positioned behind the closed door in preparation for the next trial. Each session consisted of 20 trials with an intertrial interval that typically lasted between 15 and $20 \mathrm{sec}$.

Two special procedures were used on the first two odor-discrimination sessions. First, a food pellet was placed in the well beneath the $\mathrm{S}+$ prior to the beginning of each trial in order to encourage rats to displace the lid from over the wells by enabling them to smell the food hidden beneath it. Second, if a rat displaced $S-$, it was then allowed to displace $S+$ to obtain a food pellet. On all subsequent sessions, food pellets were delivered only after $\mathrm{S}+$ was displaced, and no correction was allowed. There was one exception to this procedure: If a rat consistently failed to score above $60 \%$ correct responding by Session 20, it was permitted to correct its errors for the next five sessions (Sessions 21-25); however, in contrast to the initial correction procedure used during Sessions 1 and 2, a pellet was not delivered until after the rat displaced $\mathrm{S}+$

After a rat achieved the criterion of at least 17 correct trials out of 20 on two consecutive sessions (i.e., $85 \%$ ), we altered the procedure slightly to preclude the possibility that the rats were performing cor- rectly on the basis of auditory cues associated with the placement of $\mathrm{S}+$ and $\mathrm{S}-$, rather than on the basis of odor cues. On some trials, the experimenter tapped the floor of the apparatus in front of both food wells to mimic the sound of a lid being placed over a food well before, during, and after placing $S+$ and $S-$ over the food wells. Trials on which tapping occurred were selected in a nonsystematic manner.

Finally, the task was again changed slightly during five additional sessions. A 15-sec delay was introduced between the time of placement of the $\mathrm{S}-$ and the opening of the door to expose $\mathrm{S}+$ and $\mathrm{S}-$. The time interval between when the experimenter last touched $S+$ and last touched $S$ - remained unchanged at approximately 4 sec. This aspect of the procedure was intended to determine whether rats could discriminate which lid was last touched when the intensity of the experimenter's odor had dissipated over the 15 -sec interval. We chose $15 \mathrm{sec}$ as the interval because this is one of the time delays in our DNMS experiments, and thus we could compare the effects of increasing the "delay" in the odor-discrimination task with those in our previous research.

\section{Results and Discussion}

The rats began the choice task responding at a chance level of performance. On Session 3, the first session on which the rats were not allowed to correct errors, scores averaged $55 \%$ correct and ranged between $45 \%$ to $60 \%$. Three of the 4 rats were still performing at chance ( $M=$ $49.7 \%$ correct) after 400 trials ( 20 sessions) and thus were permitted to correct errors during Sessions 21-25. All 4 rats eventually acquired the olfactory discrimination following the correction procedure. The number of trials required to reach the criterion of $85 \%$ correct ranged from 520 to 660 trials, with a mean of 605 trials. Scores averaged $90 \%$ (ranging from $85 \%$ to $95 \%$ ) correct in the final session. A repeated measures analysis of variance (ANOVA) revealed a significant improvement in correct choices from the last five sessions prior to correction training (i.e., Sessions 16-20) to the final five sessions during which rats reached criterion $[F(1,2)=618.9, p<.01]$. The tapping procedure that was introduced after the rats had mastered the discrimination had no effect on choice accuracy, indicating that the rats had not been solving the task on the basis of spatial localization of the auditory cues associated with the placement of S+ or S-.

Figure 2 shows the mean percentage of correct responses on the last five sessions with no delay between the placement of $\mathrm{S}-$ and the opening of the door to the goal arm, as well as the five sessions during which a 15 -sec delay was interposed between these events. The rats scored a mean of $86.58 \%(S E=1.15 \%)$ correct choices with no delay, but only $53.50 \%(S E=2.18 \%)$ correct responses with the 15 -sec delay. This difference in responding across conditions was statistically significant $[F(1,3)=2,793, p<.001]$. Moreover, the percentage of correct responses did not differ significantly from chance on any of the five sessions during which the 15-sec delay was in effect $(t \mathrm{~s}<1.84, p \mathrm{~s}>.2)$.

Remarkably, all the rats eventually were able to discriminate between the two lids solely on the basis of which one had been touched more recently, even though they had been touched only $4 \mathrm{sec}$ apart. However, the rats were unable to make the discrimination when a $15-\mathrm{sec}$ interval 


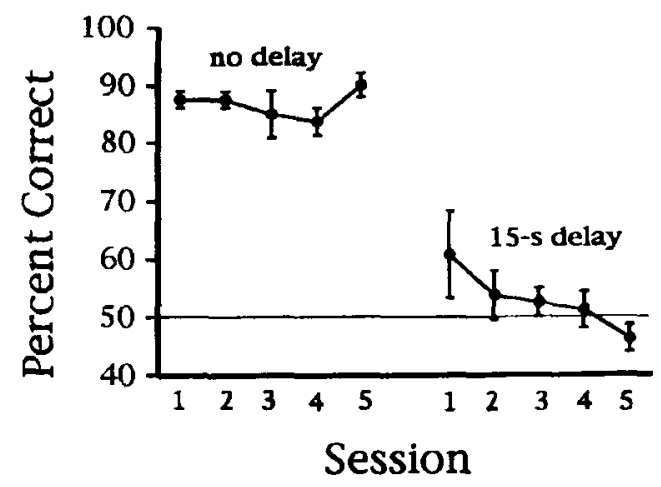

Figure 2. Performance on the odor-discrimination task over the last five sessions during which there was no delay and the following five sessions during which there was a 15-sec delay between when the experimenter last touched $S-$ and when the door was opened to expose $S+$ and $S-$. Error bars represent $S E M s$.

was interposed between when the $\mathbf{S}$ - was last touched and when they were permitted to attempt the discrimination. In view of this latter finding, it is unlikely that rats normally perform the DNMS task on the basis of a discrimination between the relative recency with which the sample and novel objects have been touched: In rat DNMS experiments, increasing the retention delay from 4 to $15 \mathrm{sec}$ has little effect on performance (Mumby et al., 1993; Mumby et al., 1995; Mumby et al., 1990; Mumby et al., 1992; Wood et al., 1993).

The most plausible way in which the rats were able to solve the present discrimination problem was on the basis of odor cues left on the lids by the experimenter. The possibility that the rats relied on visual cues from the experimenter can be discounted for two reasons. First, the doors and side walls of the apparatus were too high for the rat to see the experimenter, unless the rat was standing at the open side of the far goal area. Second, none of the rats were ever seen engaging in behavior that could be construed as looking for such visual cues.

\section{EXPERIMENT 2 Delayed Nonmatching-To-Sample}

Experiment 1 demonstrated that rats can learn to discriminate the relative recency with which two objects are handled by an experimenter when they are expressly trained to do so. Because the DNMS protocol that we have used in previous experiments (e.g., Mumby \& Pinel, 1994; Mumby et al., 1993; Mumby et al., 1995; Mumby et al., 1990; Mumby et al., 1992; Wood et al., 1993) involves a systematic difference between the sample and novel objects in terms of when they are handled by the experimenter prior to the test phase of each trial, the results of Experiment 1 raise the possibility that rats might use a similar odor-discrimination strategy to solve the DNMS task.

The purpose of Experiment 2 was to determine whether the relative recency with which the sample and novel ob- jects are handled by the experimenter influences performance of the DNMS task in rats. We compared DNMS performance under our original procedure in which the sample object always is handled more recently than is the novel object (see Figure 1) with performance under a modified procedure in which the temporal order of handling the sample and novel objects varied pseudorandomly across trials. If rats use information concerning the relative recency with which the sample and novel objects are touched by the experimenter, then rats should solve the DNMS task more easily in the original procedure.

\section{Method}

Subjects. Eight experimentally naive male Sprague-Dawley rats (7-9 weeks old) were housed and fed as in Experiment 1. Training began after the rats had been on a restricted-feeding regimen for 14 days.

Apparatus. The apparatus was the same one that had been used in Experiment 1. We used a collection of over 350 stimulus objects of various sizes, shapes, colors, and textures, similar to the "junk" objects that have been used in the monkey DNMS paradigm (see Mishkin \& Appenzeller, 1987). Each object was large enough to cover a food well but small enough and light enough to be easily displaced by a rat. No objects with obvious scents were included. The objects were washed every few sessions in a dilute solution of chlorine bleach and water.

Procedure. All testing occurred between 16 and $23 \mathrm{~h}$ after the rat's most recent meal, during the light phase of the light:dark cycle. Each rat was tested no more than once per day and no fewer than five times per week.

After being habituated to the apparatus (see Mumby et al., 1990), all rats were trained on a simple two-choice object-discrimination task. The main purpose of the object-discrimination training was to teach the rats that they could displace objects from over the food wells to receive a food pellet. The procedures for the object-discrimination training have been previously described in detail (see Mumby et al., 1990). Briefly, the same two objects served as S + and S- on all object-discrimination trials for each rat. Other than the fact that $\mathrm{S}+$ and $\mathrm{S}$ - differed in their appearance, the only way in which the objectdiscrimination procedure differed from that of the odor-discrimination procedure of Experiment 1 was that there were no systematic differences between $S+$ and $S-$ in terms of when they were handled by the experimenter. There were 25 trials per session. Training continued until each rat reached a criterion of at least 22 correct trials out of 25 on two consecutive sessions. The two objects were washed in a solution of chlorine bleach and water after every session.

DNMS training began after the rats reached the criterion on the object-discrimination problem. The rats were assigned randomly to one of two groups of 4 rats each. Groups differed slightly in terms of the details of their DNMS testing procedures. Rats in Group O (original) were tested with our original DNMS procedure (Mumby et al., 1990), which is illustrated in Figure 1. For these rats, the sample always was the most recently handled object prior to the test phase of each trial. Group R (random) was tested under a procedure in which the handling of the sample and novel object prior to the test phase varied pseudorandomly from trial to trial. Instead of positioning the novel object before the beginning of the trial, the experimenter waited until a rat had displaced the sample object in the sample phase and then quickly positioned both the sample object and the novel object over food wells at the other end of the apparatus. The temporal sequence in which the two objects were positioned varied from trial to trial according to a pseudorandom pattern. Accordingly, on half of the trials, the novel object was the last to be touched by the experimenter; on the other half of the trials, the sample was the last to be touched. For both groups of rats, the interval between when each of the two objects was last touched was approximately $4 \mathrm{sec}$, 

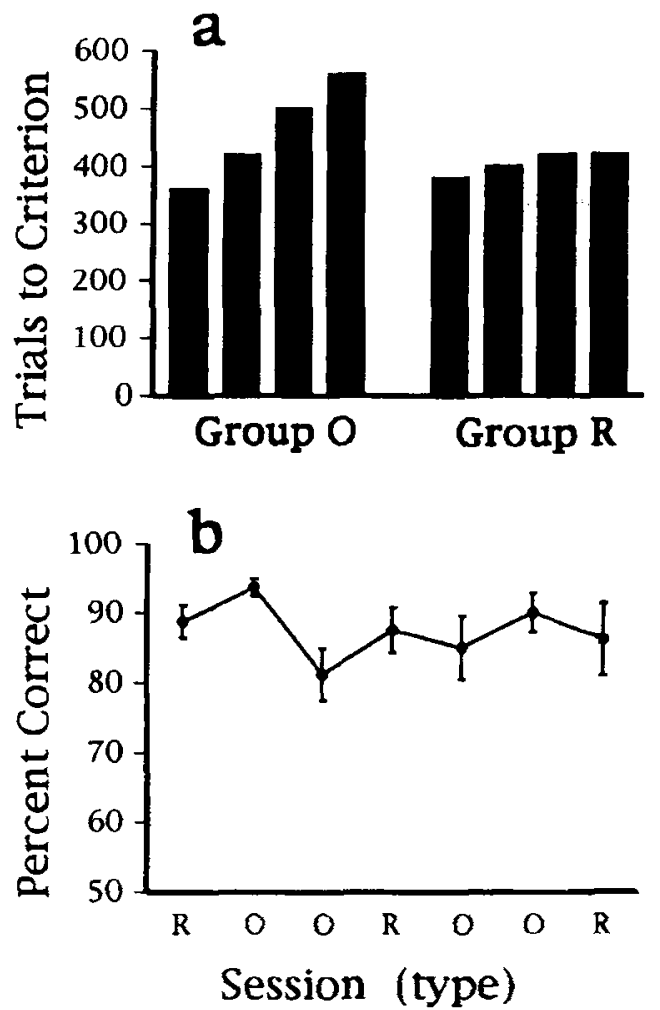

Figure 3. (a) Number of trials required to reach the DNMS performance criterion by each of the rats in Experiment 1. The 4 rats in Group $O$ were trained with the original procedure, in which the sample object was always bandled most recently prior to the test phase of each DNMS trial. The 4 rats in Group $R$ were tested in a modified procedure in which the temporal order of handling the sample and novel object prior to the test phase varied pseudorandomly across trials. (b) Asymptotic performance by the 4 rats in Group $O$ when tested with the original procedure (i.e., with the sample object always touched more recently; sessions denoted by an "O") versus with the procedure in which the temporal order of handling sample and novel objects varied pseudorandomly across trials (sessions denoted by an "R"). Error bars represent SEMs.

which was approximately equal to the interval in previous DNMS experiments (Mumby et al., 1990) and in the odor-discrimination task of Experiment 1 .

There were 20 DNMS trials, with an intertrial interval of approximately $20 \mathrm{sec}$, per session. Each rat was permitted to correct its errors during the first two DNMS sessions but not thereafter. Training for each rat continued until it reached a performance criterion of at least 17 correct trials out of 20 (i.e., $85 \%$ ) on two consecutive sessions.

Group $O$ received seven additional DNMS sessions after reaching criterion to further examine whether the rats used odor cues to solve the task. On the first, fourth, and seventh of these sessions, the procedures were identical to those that had been used to train the rats in Group R (i.e., the order in which the two objects were last touched by the experimenter varied pseudorandomly across trials). On the second, third, fifth, and sixth of these sessions, the procedures were the same as they had been during the original training (i.e., the sample object was always touched last, prior to the test phase).

\section{Results and Discussion}

The 8 rats required an average of $57.5(S E=7.96)$ trials to reach the criterion of at least 22 correct trials out of
25 on two consecutive object-discrimination sessions. This outcome was well within the range observed in previous experiments (Mumby et al., 1993; Mumby et al., 1995, Mumby et al., 1990; Mumby et al., 1992; Wood et al., 1993). However, the rats required considerably fewer trials to learn the present object-discrimination problem, which was based on the visual features of objects, than did the rats exposed to the odor-based discrimination in Experiment 1.

Figure 3 shows results from the DNMS task. The number of trials to reach the DNMS criterion for each subject in both groups is shown in panel a. All rats learned the DNMS task regardless of training condition. Rats in Group $O$ required an average of 460 trials $(S E=44.0$ ) to achieve the criterion; rats in Group $R$ required an average of 405 trials $(S E=9.6)$. This slight difference in criterion performance between groups is in a direction opposite to that predicted by an olfactory solution to the DNMS task. Mean trials to criterion did not differ significantly between groups $[t(3)=1.22, p>.25]$. Thus, in learning the DNMS task, rats in Group O did not benefit from the sample object being touched more recently than the novel object on every trial.

Panel $b$ in Figure 3 shows the effect of switching Group $O$ between $O$ and $R$ procedures after it had reached criterion performance. The percentage of correct responses did not vary across sessions $[F(1,3)<1]$ regardless of whether the sample object was always touched last (Procedure $O$ ) or whether the sample and novel objects were each touched last on half of the trials (Procedure $R$ ). This outcome indicates that, even if the rats in Group O could discriminate the relative recency with which the sample and novel objects were touched they did not rely on this information to perform the DNMS task.

\section{GENERAL DISCUSSION}

The major finding of Experiment $I$ was that, under certain conditions, rats can discriminate the relative recency with which two objects are toushed by an experimenter, even when the intertouch interval is only $4 \mathrm{sec}$. All of the rats in Experiment 1 were able to consistently make such a discrimination. However, the results of Experiments 1 and 2 suggest that rats do not solve the DNMS task by making such an olfactory discrimination. Four different observations support this conclusion.

First, the rats mastered the DNMS task in Experiment 2 in about the same number of trials regardless of whether they were trained with our original procedure, in which the sample object was always handled last prior to the test phase of every trial ( $M=460$ trials), or with a modified procedure, in which the sample object was handled last on half of the trials and the novel object was handled last on the other half of the trials ( $M=405$ trials). The rate at which the rats in the two conditions achieved the DNMS performance criterion is similar to that reported in previous experiments (Mumby \& Pinel, 1994; Mumby et al., 1993; Mumby et al., 1995; Mumby et al., 1990; Mumby et al., 1992; Wood et al., 1993). 
Second, the performance of rats in Experiment 2 that were trained with the original procedure was unaffected when the procedure was changed to one in which the sample object was the last to be touched on only half of the trials.

Third, the rats in Experiment 1 required many more trials to reach the performance criterion on the odordiscrimination task that was based on the relative recency with which two objects were touched ( $M=605$ trials) than the rats in Experiment 2 required to reach the same criterion on the DNMS task. Furthermore, 3 out of 4 rats solved the odor discrimination only after they were given 100 additional correction trials. The rats in Experiment 2 mastered the DNMS task without the benefit of similar correction trials.

Finally, although the rats were eventually able to master the odor discrimination when there was no delay between when the $S-$ was last touched and when the rats were permitted to attempt the discrimination, their performance dropped to chance levels when a 15-sec delay was introduced; presumably, the difference between the intensity of the experimenter's odor that remained on the two objects became imperceptible to the rats after $15 \mathrm{sec}$. In contrast, increasing the retention delay from 4 to $15 \mathrm{sec}$ has little effect on rats' DNMS performance (e.g., Mumby \& Pinel, 1994; Mumby et al., 1993; Mumby et al., 1995; Mumby et al., 1990; Wood et al., 1993), which suggests that they were not solving that task by discriminating the intensity of the experimenter's odor on the sample and novel objects.

In consideration of rats' well-developed olfactory system, certain memory paradigms that are ostensibly visually guided, such as the radial-arm maze, have previously come under close scrutiny for the role that odor cues might play in rats' ability to solve them (e.g., Buresova \& Bures, 1981; Roullet, Lassalle, \& Jegat, 1993). The present findings indicate that rats do not use experimenterodor cues to solve our object DNMS task. However, the rats in Experiment 1 were eventually able to discriminate the relative recency with which two objects were touched. The latter finding indicates the importance of carefully controlling for odor cues to ensure that they do not confound the intended sensory-processing demands of objectmemory tasks. Obvious precautions that the experimenters should take include washing their hands between test sessions and after placing a rat into the apparatus at the beginning of a session, minimizing the amount that they handle the objects, and making sure that there are no systematic differences between choice stimuli in terms of how much, or how recently, they were handled. For tasks that involve both a sample phase and a test phase on each trial, such as DNMS or delayed matching-to-sample, the possibility of an olfactory solution could be eliminated by using two identical objects as the sample on each trialone would serve as the sample on the sample phase, the other would serve as the sample on the test phase, and all of the objects could be positioned prior to the beginning of the trial. We recently began conducting our DNMS test- ing in this fashion, and, so far, we have found that rats are able to perform at levels that are comparable to those achieved by rats tested with our original procedure (i.e., with a single sample object, which is always handled more recently prior to the test phase). For example, in the report describing our original procedure (Mumby et al., 1990), rats' asymptotic mean score at a 15 -sec retention delay was $91 \%$ (in preliminary experiments using our modified procedure with two identical objects as the sample, asymptotic scores at a 15 -sec delay averaged about $90 \%$ ). Although this clearly indicates that rats can solve the DNMS task without the use of experimenter-odor cues, the observation that the rats in the present experiment were able to solve an experimenter-odor-based discrimination demonstrates the importance of controlling for such potential confounds when testing rats' memory for the visual features of objects.

The objects that are used as stimuli also have a role in determining how likely it is that experimenter-odor cues will confound an object-memory test. Although rats are capable of discriminating touch-produced experimenterodor cues, they might favor the use of visual cues to perform object-memory tasks if differences in the visual features of the objects are more salient than are differences in the touch-produced cues. Presumably, this was the case for the rats that learned the DNMS task. We included in our stimulus pool only objects that we considered to be highly distinctive in terms of their visual features, including size, shape, color, brightness, and intrinsic patterns. In contrast, the objects that were used for the odor discrimination in Experiment 1 were virtually identical in terms of their visual features. Although the present findings suggest that experimenter-odor cues can be the basis upon which rats solve an object-discrimination problem, they also suggest that these cues will not be especially salient in cases where the object stimuli can be easily discriminated on the basis of other sensory features.

\section{REFERENCES}

Burešová, O., \& Burěs, J. (1981). Role of olfactory cues in the radial maze performances of rats. Behavioral Brain Research, 3, 405-409.

Mishinin, M., \& Appenzeller, T. (1987, June). The anatomy of memory. Scientific American, 256, 80-89.

Mumby, D. G., \& PINEL, J. P. J. (1994). Rhinal cortex lesions and object recognition in rats. Behavioral Neuroscience, 108, 11-18.

Mumby, D. G., Pinel, J. P. J., \& Anzarut, D. S. (1991). A test battery for assessing nonspatial memory in brain-damaged rats. Society for Neuroscience Abstracts, 17, 130.

Mumby, D. G., Pinel, J. P. J., \& Dastur, F. N. (1993). Mediodorsal thalamic lesions and object recognition in rats. Psychobiology, 21, 27-36.

Mumby, D. G., Pinel, J. P. J., Kornecook, T. J., SheN, M. J., \& Redila, V. A. (1995). Memory deficits following lesions of hippocampus or amygdala in rat: Assessment by an object-memory test battery. Psychobiology, 23, 26-36.

Mumby, D. G., Pinel, J. P. J., \& Wood, E. R. (1990). Nonrecurringitems delayed nonmatching-to-sample in rats: A new paradigm for testing nonspatial working memory. Psychobiology, 18, 321-326.

Mumby, D. G., WoOd, E. R., \& PineL, J. P. J. (1992). Object-recognition memory is only mildy impaired in rats with lesions of the hippocampus and amygdala. Psychobiology, 20, 18-27. 
Otto, T., \& Eichenbalm, H. (1992). Neuronal activity in the hippocampus during delayed non-match to sample performance in rats: Evidence for hippocampal processing in recognition memory. Hippocampus, 2, 323-334.

Roullet, P., Lassalle, J. M., \& Jegat, R. (1993). A study of behavioral and sensorial bases of radial maze learning in mice. Behavioral \& Neural Biology, 59, 173-179.

Woon, E. R., Mumby, D. G., Pinel, J. P. J., \& Phillips, A. G. (1993). Impaired object recognition memory in rats following ischemiainduced damage to the hippocampus. Behavioral Neuroscience, 107, $51-62$.
Zola-Morgan, S., SQuire, L. R., \& AMaral, D. G. (1989). Lesions of the amygdala that spare adjacent cortical regions do not impair memory or exacerbate the impairment following lesions of the hippocampal formation. Journal of Neuroscience, 9, 1922-1936.

(Manuscript received December 16, 1993; revision accepted for publication October 24,1994 .) 